\title{
Kinematic Analysis and Optimization of a Wheel Loader Mechanism
}

Stefan Segla

Faculty of Mechanical Engineering, Technical University of Košice. Letná 9, 42000 Košice. Slovak Republic. E-mail: stefan.segla@tuke.sk

The paper deals with kinematic analysis and dimensional optimization of a wheel loader mechanism. The kinematic analysis is based on the kinematic constraints of all joints in the mechanism. The objective function expresses the average angular displacement of the loader bucket and the optimization process leads to its minimum value. The wheel loader mechanism contains two hydraulic drives. The first drive is ensuring rotation of the boom (lifting the bucket) and the other rotation of the bucket. Optimization of the geometric dimensions of the wheel loader mechanism links gives their optimum values which ensure lifting the bucket with its negligible angular displacement carried out by just one hydraulic drive for rotation of the boom. The other hydraulic drive ensuring rotation of the bucket is stopped during lifting the bucket. The optimization results confirmed the possibility of lifting the bucket with just one hydraulic drive for rotation of the boom.

Keywords: wheel loader, mechanism, kinematic analysis, constraint, optimization

\section{Introduction}

Wheel loaders, Fig. 1, belong to the most spread mobile working machines. They are used for excavation works and extraction of raw materials. Most of the wheel loaders produced nowadays have an integrated electronic corrective regulating system maintaining the horizontal position of the bucket during its lifting by means of the hydraulic drive carrying out rotation of the bucket.

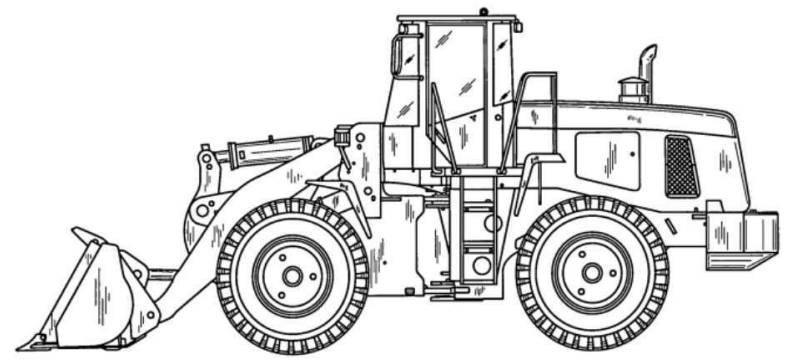

Fig. 1 Wheel loader

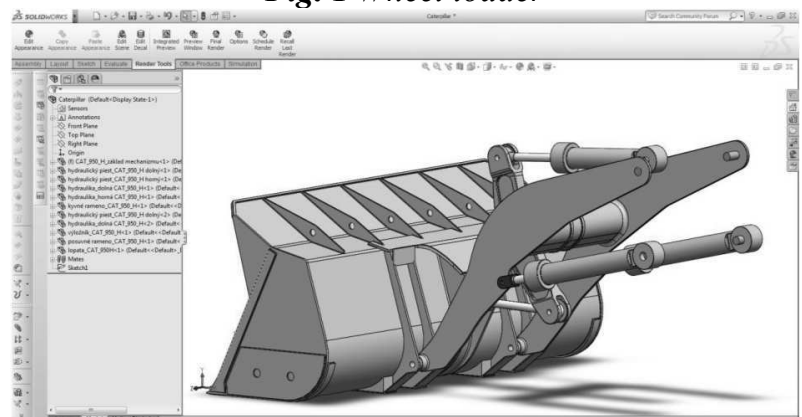

Fig. 2 Wheel loader mechanism modelled in the computer software SolidWorks

In the paper the method of kinematic constraints of all joints in the loader mechanism, Fig. 2, is used to set up its mathematical model. Numerical solution of this model leads to determination of the positions of the mechanism's links and geometrical points. This mathematical model is used for numerical optimization ([1] to [5], [7] and [8]) of the geometrical parameters of the wheel loader mechanism links by means of the MATLAB Optimization Toolbox [6].

\section{Kinematic analysis}

Fig. 3 represents scheme of the wheel loader mechanism. The task of the kinematic position analysis is to determine the relative rotational positions of the bucket 5 , Fig. 3, during its lifting by means of the hydraulic drive 6 , while the distance of the drive's 7 end points $\overline{\mathrm{EH}}$ is constant. This distance is determined in the lower (initial) position of the boom 2 so that the angular coordinate $\varphi_{5}$, determining the angular positioon of the bucket 5 , ensures its horizontal position.

The mechanism under investigation, with its kinematic scheme in Fig. 4, has two degrees of freedom (DOF) with the input motions from the two hydraulic drives 6 and 7. However, in the position kinematic analysis below, the angular coordinate $\varphi_{2}$, determining angular position of the boom 2, will be considered as the only input motion. As it was written above, the distance $\overline{\mathrm{EH}}$ of the end points of the drive 7 is constant and determined in the lower (initial) position of the boom 2 .

As the optimization variables of the problem the following geometrical dimensions are chosen:

$$
\begin{aligned}
& x_{1} \equiv \overline{\mathrm{GD}} \\
& x_{2} \equiv \overline{\mathrm{DC}} \\
& x_{3} \equiv \Varangle \gamma \\
& x_{4} \equiv \overline{\mathrm{CE}} \\
& x_{5} \equiv \overline{\mathrm{JC}} \\
& x_{6} \equiv \overline{\mathrm{BG}} \\
& x_{7} \equiv \overline{\mathrm{AJ}}
\end{aligned}
$$

the length of the link 4 (linkage), the length of the lower part of the link 3 (bucket bellcrank), the angular displacement between the lower and upper parts of the bucket bellcrank 3 , the length of the upper part of the link 3 (bucket bellcrank), the distance determining position of the joint $\mathrm{C}$ on the boom 2 , the distance between the joints B and $\mathrm{G}$ on the bucket 5 , the distance between two points A and $\mathrm{J}$ on the boom 2 . 


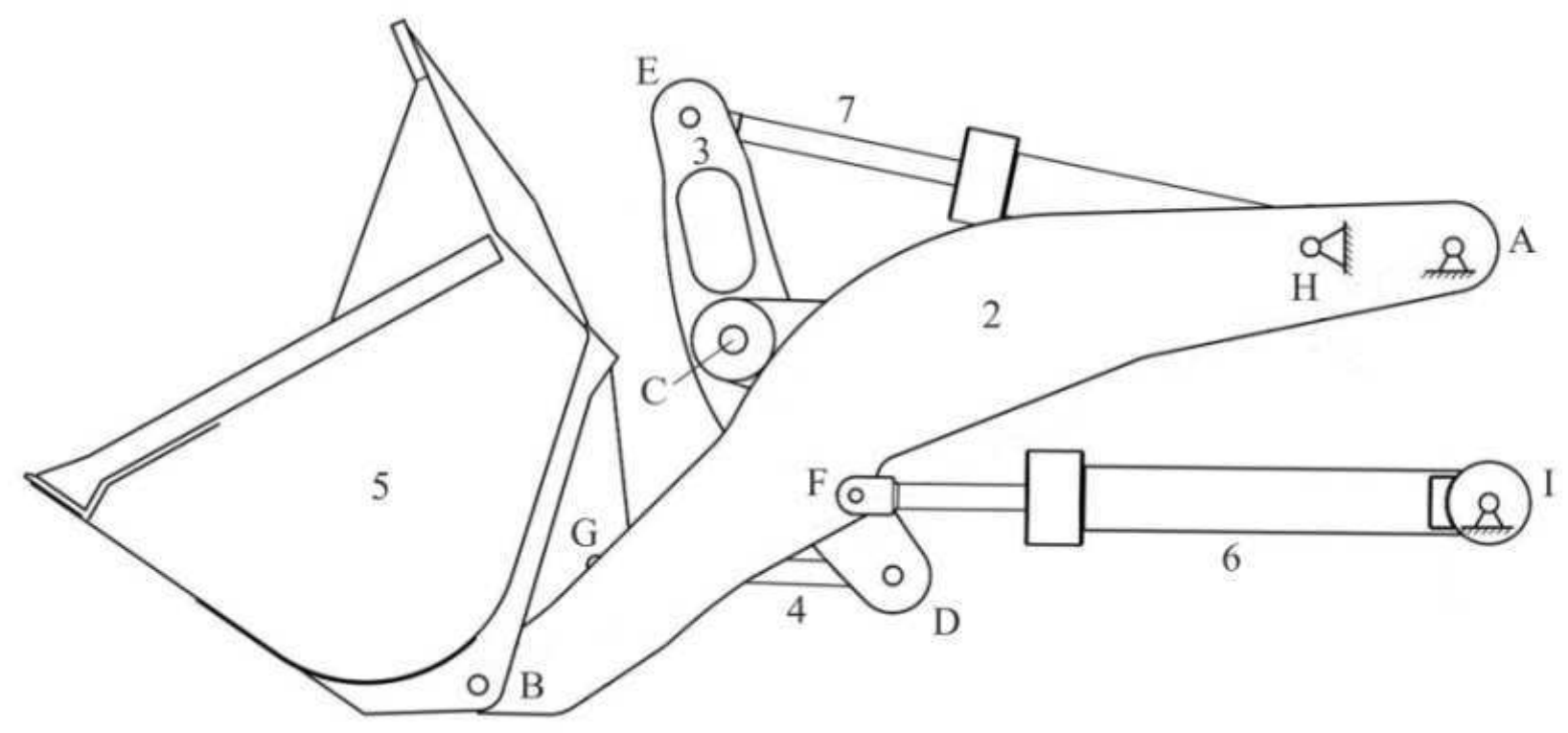

Fig. 3 Scheme of the wheel loader mechanism

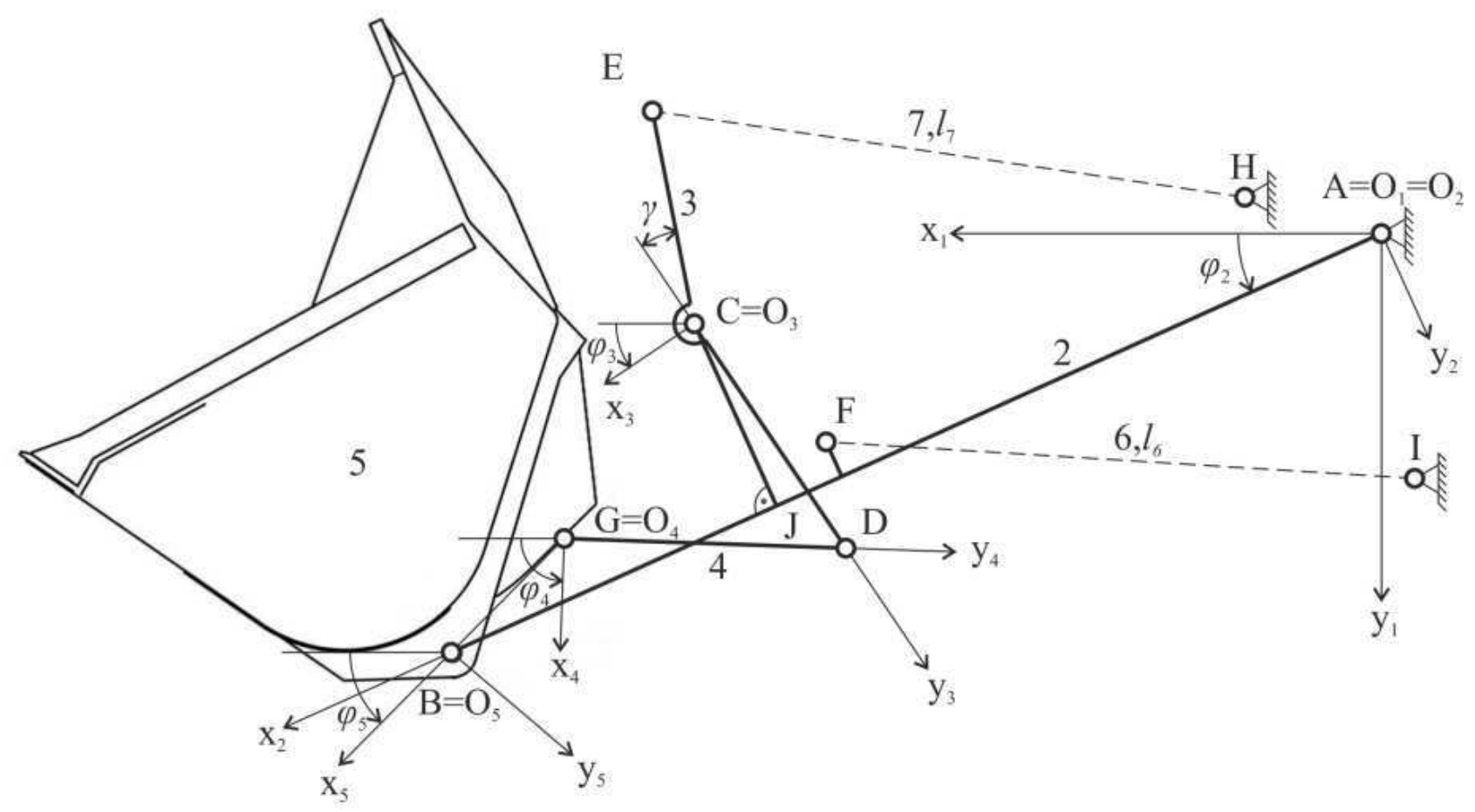

Fig. 4 Kinematic scheme of the wheel loader mechanism

Algoritmization and computer programming of this method is simpler compared to the vector method [11]. Another possibilities can be found in [14] to [16].

The configuration of the mechanical system is

$$
\mathbf{q}=\left[R_{11, x}, R_{11, y}, \varphi_{1}, R_{12, x}, R_{12, y}, \varphi_{2}, R_{13, x}, R_{13, y}, \varphi_{3}, R_{14, x}, R_{14, y}, \varphi_{4}, R_{15, x}, R_{15, y}, \varphi_{5}\right]^{\mathrm{T}},
$$

where coordinates $\varphi_{i}$ are apparent in Fig. 4 and coordinates $R_{\mathrm{ii}, x}, R_{\mathrm{ii}, y}$ determine positions of the origins of the mechanism links origins $\mathrm{O}_{\mathrm{i}}$ expressed in the ground coordinate system $\mathrm{O}_{1}, \mathrm{x}_{1}, \mathrm{y}_{1}$. described by 15 coordinates (every body is assigned an identical set of three absolute Cartesian coordinates), which can be described in a vector form as
If the global and ground coordinate systems of the link 1 are supposed to be identical, then the ground constraints are 


$$
\begin{aligned}
& R_{11, x}=0, \\
& R_{11, y}=0, \\
& \varphi_{1}=0 .
\end{aligned}
$$

The revolute joint $\mathrm{O}_{1}$ constraint (between the links 1 and 2) is

$$
\mathbf{R}_{12}=\mathbf{0} .
$$

The revolute joint $\mathrm{O}_{5}$ constraint is

$$
\mathbf{R}_{15}+\mathbf{S}_{15} \mathbf{u}_{5,50_{5}}-\mathbf{R}_{12}-\mathbf{S}_{12} \mathbf{u}_{2,2 O_{5}}=\mathbf{0}
$$

Because of $\mathbf{u}_{5,50_{5}}=\mathbf{0}$ and $\mathbf{R}_{12}=\mathbf{0}$ the following equation holds for the joint $\mathrm{O}_{5}$ constraint

$$
\mathbf{R}_{15}-\mathbf{S}_{12} \mathbf{u}_{2,20_{5}}=\mathbf{0},
$$

where the vector $\mathbf{u}_{2,2 O_{5}}$ is

$$
\mathbf{u}_{2,2 O_{5}}=\left[\begin{array}{ll}
\overline{\mathrm{AB}} & 0
\end{array}\right]^{\mathrm{T}} .
$$

The revolute joint $\mathrm{O}_{4}$ constraint is

$$
\mathbf{R}_{14}+\mathbf{S}_{14} \mathbf{u}_{4,4 O_{4}}-\mathbf{R}_{15}-\mathbf{S}_{15} \mathbf{u}_{5,5 O_{4}}=\mathbf{0},
$$

where is

$$
\mathbf{u}_{4,4 O_{4}}=\mathbf{0} \quad \text { a } \quad \mathbf{u}_{5,50_{4}}=\left[\begin{array}{ll}
-\overline{B G} & 0
\end{array}\right]^{\mathrm{T}} .
$$

The revolute joint $\mathrm{D}$ constraint is

$$
\mathbf{R}_{14}+\mathbf{S}_{14} \mathbf{u}_{4,4 D}-\mathbf{R}_{13}-\mathbf{S}_{13} \mathbf{u}_{3,3 D}=\mathbf{0},
$$

where is

$$
\mathbf{u}_{4,4 D}=\left[\begin{array}{ll}
0 & \overline{\mathrm{GD}}
\end{array}\right]^{\mathrm{T}}, \quad \mathbf{u}_{3,3 D}=\left[\begin{array}{ll}
0 & \overline{\mathrm{CD}}
\end{array}\right]^{\mathrm{T}} .
$$

The revolute joint $\mathrm{O}_{3}$ constraint is

$$
\mathbf{R}_{13}+\mathbf{S}_{13} \mathbf{u}_{3,3 O_{3}}-\mathbf{R}_{12}-\mathbf{S}_{12} \mathbf{u}_{2,2 O_{3}}=\mathbf{0},
$$

where for the vectors $\mathbf{u}_{3,30_{3}}$ and $\mathbf{u}_{2,2 O_{3}}$ the following equations hold

$$
\mathbf{u}_{3,3 O_{3}}=\left[\begin{array}{ll}
0 & 0
\end{array}\right]^{\mathrm{T}}, \quad \mathbf{u}_{2,2 O_{3}}=[\overline{\mathrm{AJ}}-\overline{\mathrm{JC}}]^{\mathrm{T}} .
$$

Because of zero vectors $\mathbf{u}_{3,30_{3}}$ and $\mathbf{R}_{12}$, eq. (11) is reduced to the form

$$
\mathbf{R}_{13}-\mathbf{S}_{12} \mathbf{u}_{2,2 O_{3}}=\mathbf{0} .
$$

The loader mechanism has 2 DOF. Therefore two further constraints formed by hydraulic drives are needed. Velocities as well as accelerations will not be needed.

The constraint generated by the drive 6 can be formed by two ways. The simpler way will be used here which does need to take into account the length of the drive. The input motion of the boom 2 starts from $\varphi_{\min }$ (the initial boom position) and stops at $\varphi_{\max }$ (the final boom position), where $\varphi=(\pi / 2)-\varphi_{2}$, with the step $\Delta \varphi$. Using the angular coordinate $\varphi$ instead of $\varphi_{2}$ is more illustrative (see Fig. 4). Mathematically this constraint is expressed in the way

$$
\varphi-\varphi_{k}=0,
$$

where $\varphi_{k}$ is expressing the constant value of the angular coordinate $\varphi$ in the interval from $\varphi_{\min }$ to $\varphi_{\max }$.

Another constraint is expressing the constant length of the hydraulic drive 7 which equals to the length $l_{7}$ of this drive (distance $\overline{\mathrm{EH}}$ ) in the lower position of the boom $2\left(\varphi=\varphi_{\text {min }}\right)$ for the horizontal position of the loader bucket. The length $l_{7}$ can be expressed in the form

$$
\left|\mathbf{R}_{1 E}-\mathbf{R}_{1 H}\right|=l_{7}
$$

and for the vector $\mathbf{R}_{1 E}$ holds

$$
\mathbf{R}_{1 E}=\mathbf{R}_{13}+\mathbf{S}_{13} \mathbf{u}_{3,3 E} \text {, }
$$

or in the scalar form $\left[\begin{array}{l}R_{1 E, x} \\ R_{1 E, y}\end{array}\right]=\left[\begin{array}{l}R_{13, x} \\ R_{13, y}\end{array}\right]+\left[\begin{array}{cc}\cos \varphi_{3} & -\sin \varphi_{3} \\ \sin \varphi_{3} & \cos \varphi_{3}\end{array}\right]\left[\begin{array}{l}-\overline{\mathrm{CE}} \sin \gamma \\ -\overline{\mathrm{CE}} \cos \gamma\end{array}\right]=$ $=\left[\begin{array}{l}R_{13, x}-\overline{\mathrm{CE}} \sin \gamma \cos \varphi_{3}+\overline{\mathrm{CE}} \cos \gamma \sin \varphi_{3} \\ R_{13, y}-\overline{\mathrm{CE}} \sin \gamma \sin \varphi_{3}-\overline{\mathrm{CE}} \cos \gamma \cos \varphi_{3}\end{array}\right]$

The vector $\mathbf{R}_{1 H}$ is

$$
\mathbf{R}_{1 H}=\left[\begin{array}{c}
\overline{\mathrm{AH}_{x}} \\
-\overline{\mathrm{AH}_{y}}
\end{array}\right]
$$

where $\overline{\mathrm{AH}_{x}}$ a $\overline{\mathrm{AH}_{y}}$ are expressing the $x$ - and $y$-component of point $\mathrm{H}$ in the ground coordinate system.

The last constraint can be finally written in the form

$$
\begin{aligned}
\left(R_{13, x}\right. & \left.-\overline{\mathrm{DE}} \sin \gamma \cos \varphi_{3}+\overline{\mathrm{DE}} \cos \gamma \sin \varphi_{3}-\overline{\mathrm{AH}_{x}}\right)^{2}+ \\
& +\left(R_{13, y}-\overline{\mathrm{DE}} \sin \gamma \sin \varphi_{3}-\overline{\mathrm{DE}} \cos \gamma \cos \varphi_{3}+\overline{\mathrm{AH}_{y}}\right)^{2}-l_{7}^{2}=0 .
\end{aligned}
$$


The total constraint vektor of the mechanism is

$$
\mathbf{C}(\mathbf{q}, t)=\left[\begin{array}{c}
R_{11, x} \\
R_{11, y} \\
\varphi_{1} \\
\mathbf{R}_{12} \\
\mathbf{R}_{15}-\mathbf{S}_{12} \mathbf{u}_{2,2 O_{5}} \\
\mathbf{R}_{14}-\mathbf{R}_{15}-\mathbf{S}_{15} \mathbf{u}_{5,50_{4}} \\
\mathbf{R}_{14}+\mathbf{S}_{14} \mathbf{u}_{4,4 D}-\mathbf{R}_{13}-\mathbf{S}_{13} \mathbf{u}_{3,3 D} \\
\mathbf{R}_{13}-\mathbf{S}_{12} \mathbf{u}_{2,20_{3}} \\
\varphi_{2}-\varphi_{2 k} \\
\left(R_{13, x}-\overline{\mathrm{DE}} \sin \gamma \cos \varphi_{3}+\overline{\mathrm{DE}} \cos \gamma \sin \varphi_{3}-\overline{\mathrm{AH}}\right)^{2}+ \\
+\left(R_{13, y}-\overline{\mathrm{DE}} \sin \gamma \sin \varphi_{3}-\overline{\mathrm{DE}} \cos \gamma \cos \varphi_{3}+\overline{\mathrm{AH}}\right)^{2}-l_{7}^{2}=0
\end{array}\right]=\mathbf{0},
$$

This vector contains 15 scalar constraints with equal number of the unknown coordinates defined by eq. (1).
For the iterative solution of the vector eq. (20) NewtonRaphson method can be used.

The constraint Jacobian matrix is derived in the form

$$
\mathbf{C}_{\mathbf{q}}=\left[\begin{array}{ccccccccccccccc}
1 & 0 & 0 & 0 & 0 & 0 & 0 & 0 & 0 & 0 & 0 & 0 & 0 & 0 & 0 \\
0 & 1 & 0 & 0 & 0 & 0 & 0 & 0 & 0 & 0 & 0 & 0 & 0 & 0 & 0 \\
0 & 0 & 1 & 0 & 0 & 0 & 0 & 0 & 0 & 0 & 0 & 0 & 0 & 0 & 0 \\
0 & 0 & 0 & 1 & 0 & 0 & 0 & 0 & 0 & 0 & 0 & 0 & 0 & 0 & 0 \\
0 & 0 & 0 & 0 & 1 & 0 & 0 & 0 & 0 & 0 & 0 & 0 & 0 & 0 & 0 \\
0 & 0 & 0 & 1 & 0 & C_{6,6} & 0 & 0 & 0 & 0 & 0 & 0 & 1 & 0 & 0 \\
0 & 0 & 0 & 0 & 1 & C_{7,6} & 0 & 0 & 0 & 0 & 0 & 0 & 0 & 1 & 0 \\
0 & 0 & 0 & 0 & 0 & 0 & 0 & 0 & 0 & 1 & 0 & 0 & -1 & 0 & C_{8,15} \\
0 & 0 & 0 & 0 & 0 & 0 & 0 & 0 & 0 & 0 & 1 & 0 & 0 & -1 & C_{9,15} \\
0 & 0 & 0 & 0 & 0 & 0 & -1 & 0 & C_{10,9} & 1 & 0 & C_{10,12} & 0 & 0 & 0 \\
0 & 0 & 0 & 0 & 0 & 0 & 0 & -1 & C_{11,9} & 0 & 1 & C_{11,12} & 0 & 0 & 0 \\
0 & 0 & 0 & 0 & 0 & C_{12,6} & 1 & 0 & 0 & 0 & 0 & 0 & 0 & 0 & 0 \\
0 & 0 & 0 & 0 & 0 & C_{13,6} & 0 & 1 & 0 & 0 & 0 & 0 & 0 & 0 & 0 \\
0 & 0 & 0 & 0 & 0 & 1 & 0 & 0 & 0 & 0 & 0 & 0 & 0 & 0 & 0 \\
0 & 0 & 0 & 0 & 0 & 1 & C_{15,7} & C_{15,8} & C_{15,9} & 0 & 0 & 0 & 0 & 0 & 0
\end{array}\right],
$$

where

$$
\begin{gathered}
C_{6,6}=\overline{\mathrm{AB}} \cos \varphi_{2}, \quad C_{7,6}=-\overline{\mathrm{AB}} \sin \varphi_{2}, \quad C_{12,6}=-\overline{\mathrm{AC}} \sin \varphi_{2}-\overline{\mathrm{CD}} \cos \varphi_{2}, \\
C_{13,6}=\overline{\mathrm{AC}} \cos \varphi_{2}-\overline{\mathrm{CD}} \sin \varphi_{2}, \quad C_{10,9}=\overline{\mathrm{DF}} \cos \varphi_{3}, \quad C_{11,9}=\overline{\mathrm{DF}} \sin \varphi_{3}, \\
C_{10,12}=-\overline{\mathrm{GF}} \cos \varphi_{4}, \quad C_{11,12}=-\overline{\mathrm{GF}} \sin \varphi_{4}, \quad C_{8,15}=\overline{\mathrm{BG}} \sin \varphi_{5}, \quad C_{9,15}=-\overline{\mathrm{BG}} \cos \varphi_{5}, \\
C_{15,7}=2\left(R_{13, x}-\overline{\mathrm{DE}} \sin \gamma \cos \varphi_{3}+\overline{\mathrm{DE}} \cos \gamma \sin \varphi_{3}-\overline{\mathrm{AM}}\right), \\
C_{15,8}=2\left(R_{13, y}-\overline{\mathrm{DE}} \sin \gamma \sin \varphi_{3}-\overline{\mathrm{DE}} \cos \gamma \cos \varphi_{3}+\overline{\mathrm{MI}}\right), \\
C_{15,9}=2\left(R_{13, x}-\overline{\mathrm{DE}} \sin \gamma \cos \varphi_{3}+\overline{\mathrm{DE}} \cos \gamma \sin \varphi_{3}-\overline{\mathrm{AM}}\right) \overline{\mathrm{DE}}\left(\sin \gamma \sin \varphi_{3}+\cos \gamma \cos \varphi_{3}\right)+ \\
+2\left(R_{13, y}-\overline{\mathrm{DE}} \sin \gamma \sin \varphi_{3}-\overline{\mathrm{DE}} \cos \gamma \cos \varphi_{3}+\overline{\mathrm{MI}}\right) \overline{\mathrm{DE}}\left(\sin \gamma \cos \varphi_{3}+\cos \gamma \sin \varphi_{3}\right)
\end{gathered}
$$

If the constraint Jacobian matrix (21) is nonsingular then the first order approximation gives

$$
\mathbf{C}_{\mathbf{q}}\left(\mathbf{q}_{i}, t\right) \Delta \mathbf{q}_{i}=-\mathbf{C}\left(\mathbf{q}_{i}, t\right)
$$


This equation can be solved for the vector of Newton differences $\Delta \mathbf{q}_{i}$. This vector can be used to iteratively update the vector of the system coordinates as

$$
\mathbf{q}_{i+1}=\mathbf{q}_{i}+\Delta \mathbf{q}_{i}
$$

The angular coordinate $\varphi_{5}$ in eq. (1) can be used to define the more illustrative angle $\beta$ determining position of the bucket 5 in the form

$$
\beta=\pi-\varphi_{5}
$$

\section{Formulation of the optimization problem}

The optimization goal consists in achieving optimal values of the optimization variables $x_{1}$ to $x_{7}$ which ensure lifting the loader bucket by means of the hydraulic drive for rotation of the loader boom holding its horizontal position with sufficient accuracy.

Lifting the bucket will be carried out in the interval of the input motion from $\varphi_{\min }$ (the initial boom position) to $\varphi_{\max }$ (the final boom position) with the step $\Delta \varphi$. If the desired value of the bucket horizontal position is denoted by $\beta_{d}$ then the objective function can be expressed in the form

$$
f_{o p}=\frac{1}{N} \sum_{i=1}^{N} \sqrt{\left(\beta_{i}-\beta_{d}\right)^{2}}
$$

where $\mathrm{N}$ is the number of the boom positions in which the value of $\left(\beta_{i}-\beta_{d}\right)$ is evaluated in the interval of mechanism motion from $\varphi_{\min }$ to $\varphi_{\max } . \beta_{i}$ is the actual value of the bucket angular displacement in the $i$-th step determined by the value of $\varphi_{\mathrm{i}}=\varphi_{\min }+i \Delta \varphi$. The objective function (26) determines the average value of the difference $\left(\beta_{i}-\beta_{d}\right)$ in the interval from $\varphi_{\min }$ to $\varphi_{\max }$.

The optimization problem defined above does not need any restrictions (except so called natural restrictions) because the lower and upper bounds of the optimization variables are relatively closed to the geometric dimensions of the Caterpillar 950 wheel loader.

Similar optimization algorithms for optimization of various kinds of mechanisms were used in [9] and [10]. In [11] and [12] not only geometrical but also mechanical parameters of mechanisms were optimized and some restrictions were taken into account.

\section{Results of optimization}

In Table 1 the units, lower and upper bounds and optimum value of all optimization variables are given.

Tab. 1 Optimization variables and their optimal values

\begin{tabular}{|c|c|c|c|c|}
\hline Optimization variable & Units & Lower bound & Upper bound & Optimal value \\
\hline$x_{1} \equiv \overline{\mathrm{GD}}$ & $\mathrm{m}$ & 0.35 & 1.30 & 0.818906 \\
\hline$x_{2} \equiv \overline{\mathrm{DC}}$ & $\mathrm{m}$ & 0.25 & 0.95 & 0.671304 \\
\hline$x_{3} \equiv \Varangle \gamma$ & $\mathrm{rad}$ & -0.17 & 1.2 & 0.039287 \\
\hline$x_{4} \equiv \overline{\mathrm{CE}}$ & $\mathrm{m}$ & 0.09 & 0.60 & 0.335932 \\
\hline$x_{5} \equiv \overline{\mathrm{JC}}$ & $\mathrm{m}$ & 0.09 & 0.9 & 0.884922 \\
\hline$x_{6} \equiv \overline{\mathrm{BG}}$ & $\mathrm{m}$ & 0.30 & 0.55 & 0.35 \\
\hline$x_{7} \equiv \overline{\mathrm{AJ}}$ & $\mathrm{m}$ & 0.95 & 1.55 & 0.967387 \\
\hline
\end{tabular}

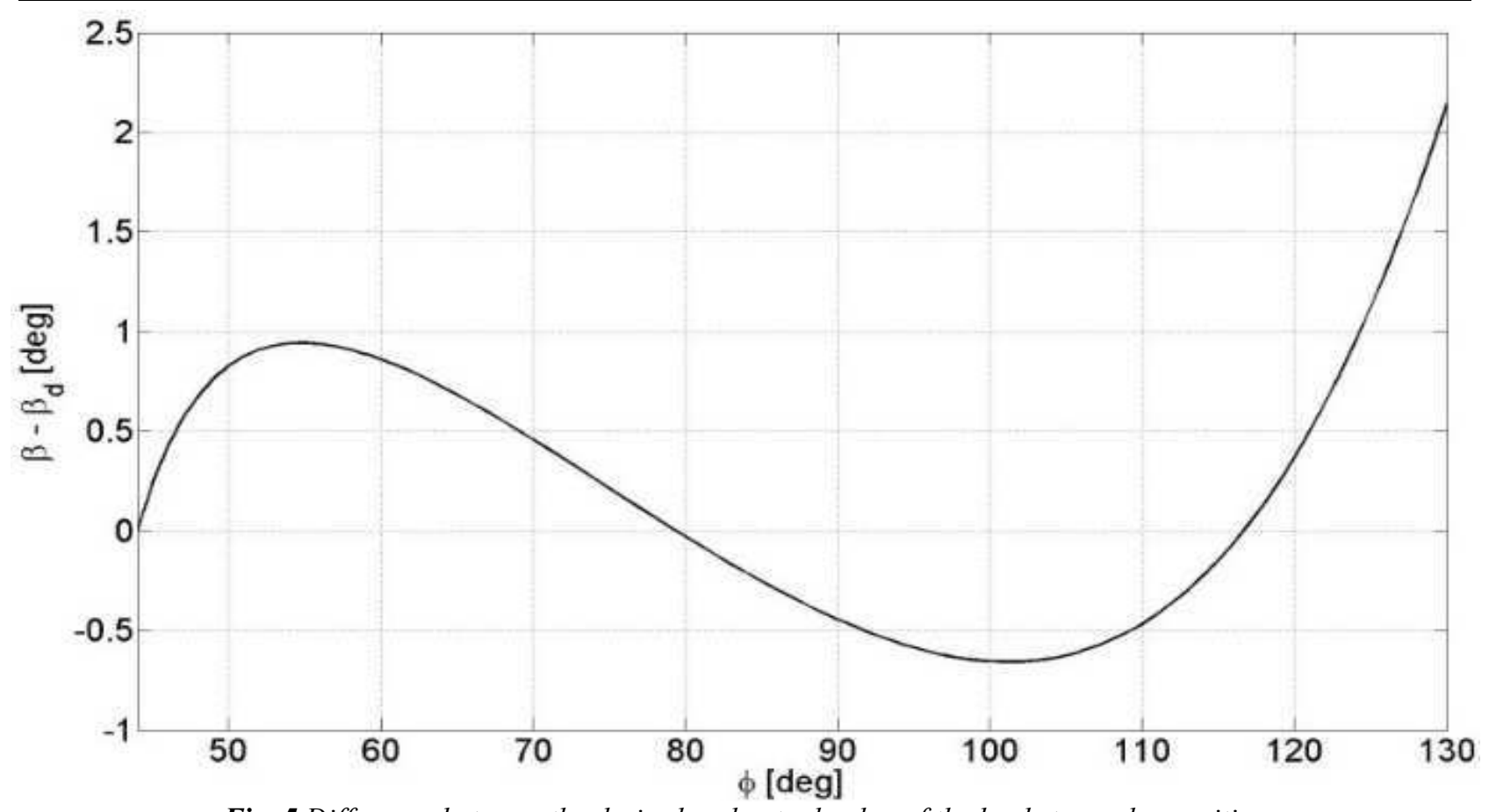

Fig. 5 Difference between the desired and actual value of the bucket angular position 
The objective function $f_{o p}$ (26) was evaluated in each optimization iteration from $\varphi=45^{\circ}$ to $\varphi=130^{\circ}$ with the step $\Delta \varphi=1^{\circ}$. The number of evaluated mechanism positions is $\mathrm{N}=86$. The results of optimization are characterized by the optimum values of the optimization variables given in Tab. 1. The value of the objective function is $f_{\text {op }}=0.012455 \mathrm{rad}\left(0.714^{\circ}\right)$ which determines the average value of the difference between the desired and actual value evaluated in the interval from $\varphi_{\min }$ to $\varphi_{\max }$. Fig. 5 shows the dependence of the difference between the desired and actual value of the bucket angular position and $\varphi$. The maximum value of the difference is in the upper bucket position $\varphi_{\max }=130^{\circ}$ and is $\left(\beta-\beta_{d}\right)_{\max }=2.17^{\circ}$. This value is fully acceptable from the practical point of view.

\section{Conclusion}

The results of optimization showed that it is possible to find such values of geometric dimensions of the wheel loader mechanism that lifting the loader bucket can be carried out by just one hydraulic drive (for rotation of the loader boom). Thanks to this fact the integrated electronic corrective regulating system maintaining horizontal position of the bucket during its lifting is not needed.

\section{Acknowledgement}

This work was supported by the grant project VEGA No. 1/0731/16.

\section{References}

[1] SEGLA, S., KALKER-KALKMAN, C.M., SCHWAB, A.L. (1998). Statical balancing of a robot mechanism with the aid of a genetic algorithm. In: Mechanism and Machine Theory, Vol. 33, pp. 163-174.

[2] KALKER-KALKMAN, C.M., SEGLA, S. (2000). Optimization and comparison of trailer suspension systems. In: Engineering Mechanics, Vol. 7, pp. 121-134.

[3] C.M., SEGLA, S., KALKER-KALKMAN (1996). Optimization of the two-wheel trailer suspension parameters. In: Engineering Mechanics, Vol. 3, pp. 177-186.

[4] KALKER-KALKMAN, C.M. (1991). Optimal design with the aid of randomization methods. In: Engineering with Computers, Vol. 7, pp. 173184.
[5] KALKER-KALKMAN, C.M. (1997). A general multi-objective optimization program for mixed continuous/integer variabels based on genetic algorithms. In: Progress in Industrial Mathematics at ECMI 97, B.G. Teubner Stuttgart, pp. 348-356.

[6] Optimization Toolbox for Use with Matlab, User's Guide Version 2 (2000). The MathWorks, Inc., Natick (USA).

[7] HANZL, P., ZITKOVÁ, I. MACH, J. (2017). Optimization of the Pressure Porous Sample and Its Manufacturability by Selective Laser Melting. Manufacturing Technology. In: Manufacturing Technology, Vol. 17, pp. 34-38.

[8] KYNCL, J., KELLNER, T., KUBIŠ, R. (2017). Tricanter Production Optimization by Digital Factory Simulation Tools. In: Manufacturing Technology, Vol. 17, pp. 49-53.

[9] SEGLA, Š., HRONCOVÁ, D., POLÁKOVÁ, Z. (2004). Optimization of statical balancing of a lift mechanism. In: Acta Mechanica Slovaca, Vol. 3C, pp. 153-160.

[10] SEGLA, Š., POLÁKOVÁ, Z., HRONCOVÁ, D. (2002). Optimization of statical balancing of a manipulator with varying loads. In: Acta Mechanica Slovaca, Vol. 6, pp. 71-80.

[11] SEGLA, S., CIUPITU, L., REICH, S. (2006). Optimization of a spring balancing mechanism for parallelogram robot mechanism. In: Journal of Mechanisms and Manipulators, Vol. 5, pp. 43-48.

[12] SEGLA, S., KALKER-KALKMAN, C.M. (2005). Optimization of torsional systems with self-regulated pneumatic clutches. In: Computer Assisted Mechanics and Engineering Sciences, Vol. 12, pp. 9-16.

[13] SHABANA,A.A. (2005). Dynamics of Multobody Systems. Cambridge University Press, Cambridge.

[14] CHALUPA, M., VEVERKA, J. (2017). Precising of vehicles handling valuation. In: Manufacturing Technology, Vol. 17, No. 3, pp. 312-319.

[15] BULÍN, R., HAJŽMAN, M., POLACH, P., ŠIKA, Z., ZAVŘEL, J. (2017). Dynamic analysisof a cable manipulator using multibody approaches. In: Manufacturing Technology, Vol. 17, No. 2, pp. 151-157.

[16] CHALUPA, M., VEVERKA, J. (2016). Handling simulation of vehicles, Vol. 16, No. 6, pp. 12641269. 